
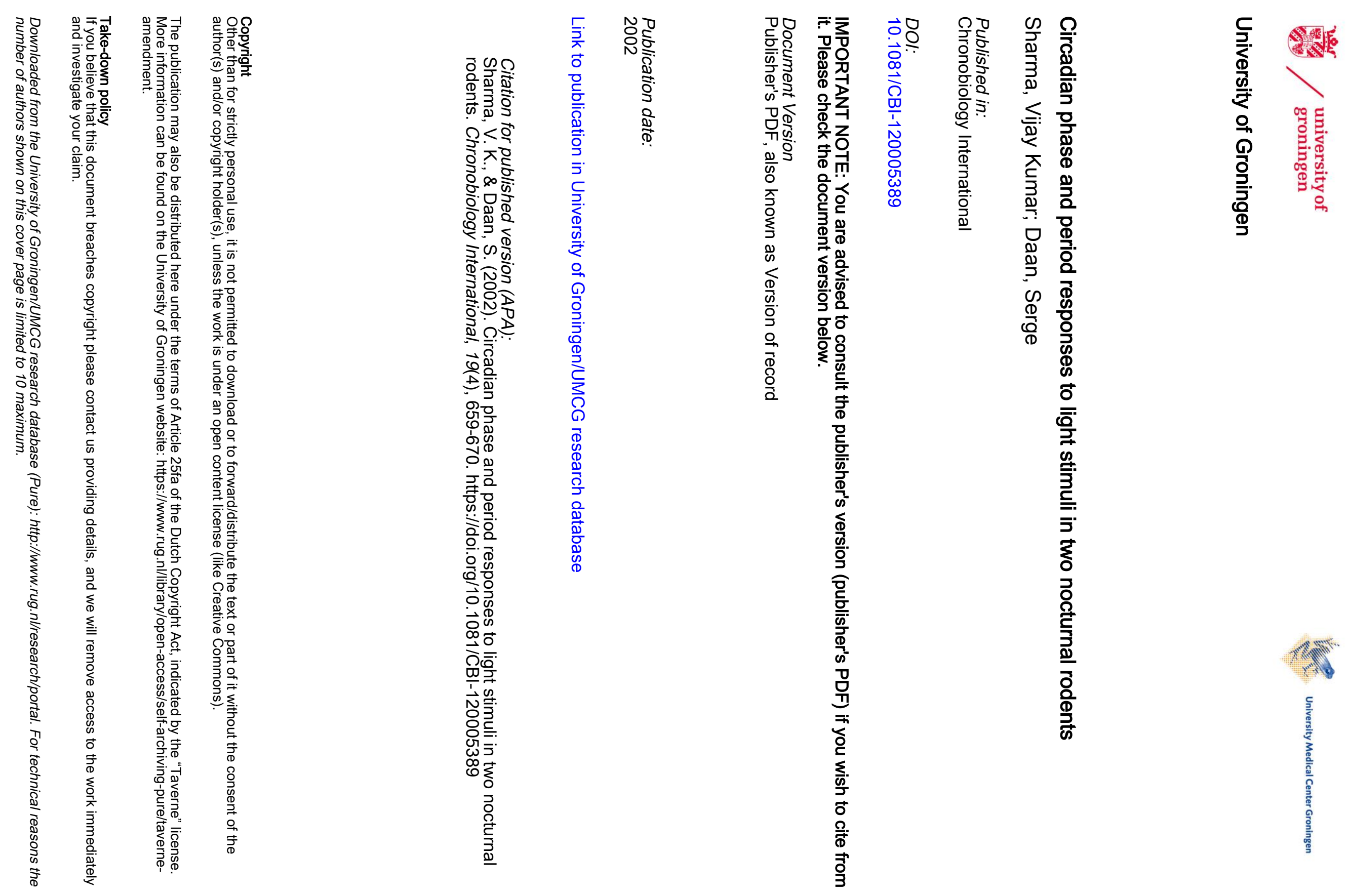
CHRONOBIOLOGY INTERNATIONAL, 19(4), 659-670 (2002)

\title{
CIRCADIAN PHASE AND PERIOD RESPONSES TO LIGHT STIMULI IN TWO NOCTURNAL RODENTS
}

\author{
Vijay Kumar Sharma ${ }^{1, *}$ and Serge Daan ${ }^{2}$ \\ ${ }^{1}$ Chronobiology Laboratory, Evolutionary and Organismal Biology \\ Unit, Jawaharlal Nehru Center for Advanced Scientific Research, \\ Jakkur, P.O. Box 6436, Bangalore 560 064, Karnataka, India \\ ${ }^{2}$ Zoological Laboratory, Biological Center, University of Groningen, \\ Kerklaan 30, P.O. Box 14, 9750 AA Haren, The Netherlands
}

\begin{abstract}
We report period response curves $(\tau \mathrm{RC})$ for two nocturnal Murid species from India, Mus booduga and Mus platythrix. We further discuss the method of phase shift estimation in the presence of $\tau$-changes, because such changes pose a serious methodological problem in the estimation of phase shifts. Although the $\tau \mathrm{RC}$ indicates that most of the phase shifts are associated with small changes in $\tau$, the period changes across all the phases showed a significant positive correlation with the phase shifts. We conclude that $\tau \mathrm{RCs}$ are a reality even in nocturnal mammals, although their amplitude is less than what is usually found in diurnal mammals, and requires a larger data set to be distinguished from noise. (Chronobiology International, 19(4), 659-670, 2002)
\end{abstract}

Key Words: Circadian; Diurnal; Nocturnal; Phase response curve; Period response curve

\footnotetext{
*Corresponding author. E-mail: vsharma@jncasr.ac.in
} 


\section{INTRODUCTION}

The entrainment of circadian systems by the Earth's rotation is primarily the result of rapid phase shifting responses to the periodic light environment. In addition, light exerts subtle effects on the endogenous circadian cycle length that may persist long after the stimulus has subsided. These "after-effects" ${ }^{[1]}$ in constant conditions have long been known to reflect properties of the prior zeitgeber, such as its period $(T)$ and light/dark ratio. They can also occur as a consequence of brief light stimuli such as used to assess phase response curves (PRCs). It has been proposed that the after-effects facilitate the fine-tuning of circadian clocks to the external periodicity. ${ }^{[2]}$ By precise tuning of the endogenously generated cycle length to $24 \mathrm{~h}$, circadian clocks would not require net phase shifts every day and enhance the accuracy of circadian pacemaker during entrainment. ${ }^{[3]}$ According to this view, the entrainment process is based on combined phase-dependent responses in both phase and period to the environmental lighting conditions. The essential prerequisite for this concept is a period response curve $(\tau \mathrm{RC})$ for brief light stimuli. Such response curves have been measured in several diurnal rodent species (Fig. 6 of Ref. [3]). These curves consistently show the same general shape as the PRC, with decelerations during the early subjective night and accelerations during the late subjective night. On theoretical grounds it has been predicted that nocturnal burrowing animals would benefit less from such $\tau$-responses than their diurnal counterparts. ${ }^{[3,4]}$ Pittendrigh and Daan (Table 4 of Ref. [2]) reported similar though minor effects of brief light stimuli on the subsequent free running period $(\tau)$ in four species of nocturnal mammals. In the Syrian hamster, Elliott (Fig. 8 of Ref. [5]) observed reductions of $\tau$ following advancing light pulses. To date, no complete $\tau \mathrm{RC}$ has been reported for a nocturnal mammal, in spite of the fact that the circadian system of nocturnal mammals has been far more extensively studied than that of diurnal mammals. The single exception is the common vole, ${ }^{[6]}$ a species that is not exclusively nocturnal but characterized by ultradian feeding activity all through the $24 \mathrm{~h}$. In this study, we report the $\tau \mathrm{RC}$ for two fully nocturnal Murid species from India, Mus booduga and Mus platythrix. In M. booduga, the PRC evoked by brief light pulses of 1000 lux intensity and 15min duration has the shape of a standard PRC with phase delays during the early subjective night and phase advances during the late subjective night. ${ }^{[7]} \mathrm{We}$ conclude that a $\tau \mathrm{RC}$ is a reality in nocturnal mammals also, but that its amplitude is less than usually found in diurnal mammals, and requires a larger data set to be distinguished from noise.

\section{EXPERIMENTAL METHODS}

Adult male field mice (M. booduga: $n=130$ and M. platythrix: $n=69)$ were captured from the fields surrounding the Madurai Kamaraj University campus $\left(9^{\circ} 58^{\prime} \mathrm{N}, 78^{\circ} 10^{\prime} \mathrm{E}\right)$. At this latitude, most days are sunny with clear skies. The animals were initially maintained under a light/dark regime (LD 12h:12h) for ca. $15 \mathrm{~d}$ and then 
introduced to cages equipped with running wheels. An eccentrically placed magnet in the running wheel enabled the recording of wheel revolutions on an Esterline Angus event recorder (A $620 \mathrm{X}$ ). Mice feed consisting of grains and water were available ad libitum. The circadian systems of the animals were allowed to free-run under constant dark (DD) conditions in a light-tight, adequately ventilated experimental room. Battery-powered torch lights with a combination of filters transmitting only red light of wavelengths exceeding $640 \mathrm{~nm}$ were used for feeding and cleaning purposes for short intervals. The activity patterns of 20 mice in separate running wheels, placed on open shelves in the experimental room, could be assayed concurrently. In this setup, we have never observed any obvious interaction between the circadian systems of animals, neither in any LD nor in DD. Temperature $\left(25 \pm 1{ }^{\circ} \mathrm{C}\right)$ and humidity $(70 \pm$ $5 \%$ ) in the room were recorded with a thermohygrograph (Wilh. Lambrecht KG, Göttingen, Germany) and were found to be nearly constant throughout the experiment.

Adult mice ( $M$. booduga) were subjected to diffuse daylight pulses of approximately 1000 lux intensity for $15 \mathrm{~min}$ duration after ca. $15 \mathrm{~d}$ of stabilized freerun in DD. The desired phases (ct) were calculated from the prior stable free-running rhythm. The light pulses were given by positioning the animals inside a room fitted with windows on its three sides, at appropriate distances from the direct sunlight. Light pulses were always restricted to the interval between 11:00 and 13:00 h in order to minimize variation in light intensity profile and changes in spectral composition of light pulse. Only sunny days with clear sky were chosen for light pulse administration in order to minimize variation in light intensity during the experiment. To set the light intensity at ca. 1000 lux, the light probe of a United Detector Technology (UDT, Texas, USA) optometer was placed inside the plexiglass box and its experimental position relative to the windows adjusted to this intensity. After $15 \mathrm{~min}$ of light exposure, cages were returned to the dark room. The experiments with M. platythrix were done using similar protocol, but the animals were exposed to incandescent light pulses of ca. 1000 lux intensity for $15 \mathrm{~min}$ duration. Free-running periods were computed using regression lines through 10-15 onsets of locomotor activity preceding the light pulse and through 10-15 onsets of activity after the light pulse, leaving the first three onsets out to let transients subside. Phase-shifts $(\Delta \phi)$ and circadian time $(\mathrm{CT})$ are expressed in circadian hours (i.e., $\times \tau / 24)$ throughout the text. Period changes $(\Delta \tau)$ are estimated by subtracting the postpulse $\tau$ from the prepulse $\tau$. Therefore, an increase in $\tau$ would result in a negative value of $\Delta \tau$ and a decrease in $\tau$ would result in positive $\Delta \tau$. An increase in $\tau$ (deceleration in $\tau$ ) can be thought to be analogous to the slowing down of circadian pacemaker and a decrease in $\tau$ (acceleration) as it's speeding up.

\section{DETERMINATION OF PHASE SHIFTS $(\Delta \phi)$ AND PERIOD CHANGES $(\Delta \tau)$}

The after-effects of single brief light pulses on $\tau$ pose a nonnegligible methodological problem in the determination of phase shifts. Before presenting 
the results, we therefore first digress on the method of phase shift measurement in the presence of $\tau$-changes.

In the classic paradigm, a phase shift of the circadian rhythm following a single perturbation - such as a brief light pulse-is measured by comparing two regressions (or eye-fitted lines) through two series of phase reference points in an actogram: before and after perturbation. The displacement of these lines relative to each other is considered to represent the phase shift. As long as the period length is the same before and after perturbation the two regression lines run parallel, and it does not make any difference on which day or time the phase shift is measured. In fact, with unchanged $\tau$ the shift persists indefinitely. In order to meet the condition of equal $\tau$, the usual practice is to start fitting the post-pulse line after several cycles to let transients subside. This is of course correct as long as the condition is met. If it is not met, the method faces the problem that the two regressions diverge and therefore it becomes important to define precisely at which date and time their position relative to each other is calculated. A common practice is to take the time of the first expected phase reference after the pulse. $\tau$ s rarely stabilized at a new value immediately following the perturbation. In fact, the transients following a light pulse typically describe gradual changes in cycle length. As Fig. 1 (upper panel) shows with a theoretical example, there is a degree of arbitrariness in assuming on which day the transients are over and the new value of $\tau$ is established. This arbitrariness affects the measurement of both $\Delta \tau$ and $\Delta \phi$. This is illustrated by drawing the regressions through the onsets on days $2-10$ and days 4-12 after the light pulse. The first case yields an intercept of -0.93 and a slope of -0.11 , thus estimating $\Delta \phi=0.93, \Delta \tau=0.11$. The day $4-12$ regression yields $\Delta \phi=1.19 \mathrm{~h}, \Delta \tau=0.07 \mathrm{~h}$. The differences underscore the importance of precisely defining the choice of regression lines.

What should then be the appropriate measurement of both phase shift and period change under these conditions? One might opt for estimating quadratic regressions of the phase reference points on sequence number beginning at consecutive phase references. This would allow us to decide that transients are over when there is no significant contribution of the squared sequence number on the explained variance around the regression. Such a procedure would yield different starting days for different actograms depending on the precision in each case. To avoid such arbitrariness, we have made an exercise based on all $(n=33)$ advancing pulses presented at ct 20 and 22, and on all $(n=44)$ delaying pulses presented at ct 14 and 16 to $M$. booduga. In each record, all activity onsets were expressed as the deviation in hours from the expected times on the basis of the prepulse regression. In Fig. 1, these relative onsets are averaged separately for the advancing and delaying pulses. Regression analysis on the postpulse events shows the following: for phase delays, squared day number does not contribute significantly to the explained variance in the onset time; hence, there is no evidence of transients. Day number itself does contribute significantly $(p<0.02)$ to this variance; hence there is a significant, although only small increase in $\tau(\Delta \tau=-0.016 \mathrm{~h})$ due to the pulse. For phase advances, both the linear 


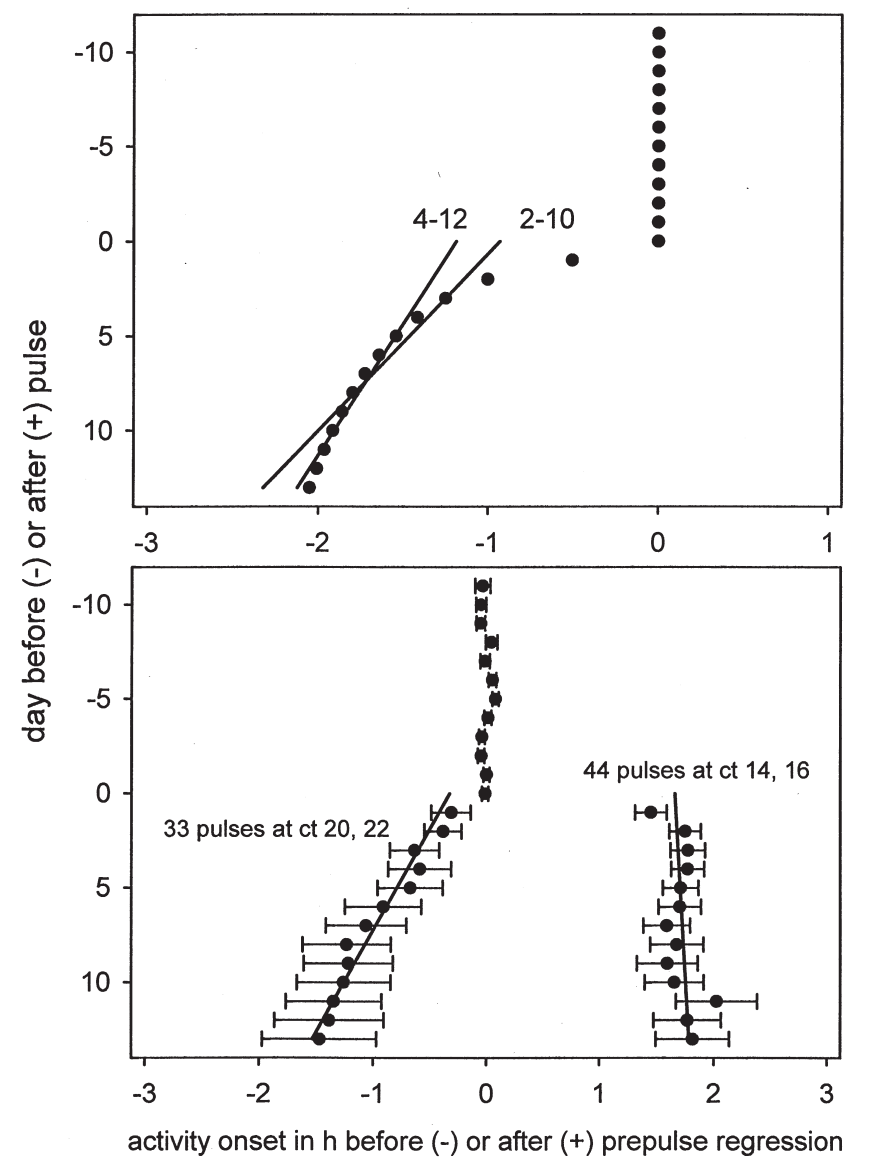

Figure 1. Upper panel: consequence of a gradual change in $\tau$ on the measurement of phase shift and $\tau$ change. In a theoretical example, transients are assumed to gradually subside following an advancing light pulse. Regressions are drawn through onsets (solid dots) in cycles 2-10 after the pulse, and cycles $4-12$, respectively. The resulting intercepts ( $=$ phase shifts) and slopes $(=\tau$ changes) are $-0.93,-1.19 \mathrm{~h}(\Delta \phi)$ and $-0.11,-0.07 \mathrm{~h}(\Delta \tau)$, respectively. Lower panel: calculation of the average $\tau$ change and phase shift following 33 light pulses at ct 20 and 22, and following 44 light pulses at ct 14 and 16 in M. booduga. In each individual, the prepulse regression was calculated and all onsets before and after the pulse calculated as deviation from this regression. Prepulse deviations are averaged for all 77 data sets, postpulse deviations for the advancing and delaying pulses separately. Horizontal error bars represent 1 SEM at both sides of the mean. Oblique lines represent postpulse regressions through deviations following advancing and delaying pulses computed over days $3-11$.

$(p<0.001)$ and quadratic components $(p<0.02)$ are significant; hence there is evidence of transient cycles. We consecutively omitted days $1,2, \ldots$ from this analysis, and established that the quadratic component becomes insignificant from day 3 onwards. Hence, we decided to analyze phase shifts and $\tau$-changes on the basis of postpulse regressions starting with the fourth onset following the light 
pulse to eliminate any transient cycles appearing after the light pulses. For the average data in Fig. 1 the slope is -0.084 , meaning that the $\Delta \tau$ is $+0.084 \mathrm{~h}$. The intercept of the regression on day 0 is -0.903 , yielding an estimate for the advance phase shift of +0.903 .

\section{RESULTS}

\section{Mus booduga}

The average PRC of M. booduga has the shape of a standard PRC, with large phase delays in the early subjective night and large phase advances in the late subjective night (Fig. 2A reported in Ref. [7]). The PRC has no region that can be described as a true "dead-zone." Figure 2B shows the $\tau$-changes, recorded, following the light pulses. The $\tau$-changes can be of considerable size-up to $0.7 \mathrm{~h}$ - and they occur in a phase-dependent manner. Period lengthening was observed in animals exposed to light pulses during the early subjective night (ct 12-18) and period shortening was observed during the late subjective night (ct 20-24). The period changes across all the phases showed a significant positive correlation with the phase shifts $(r=+0.44, p<0.0001$; Fig. 3 ). The average $\tau$ of the locomotor activity rhythm of the animals used in this experiment was $23.28 \pm$ $0.44 \mathrm{~h}($ mean $\pm \mathrm{SD})$.

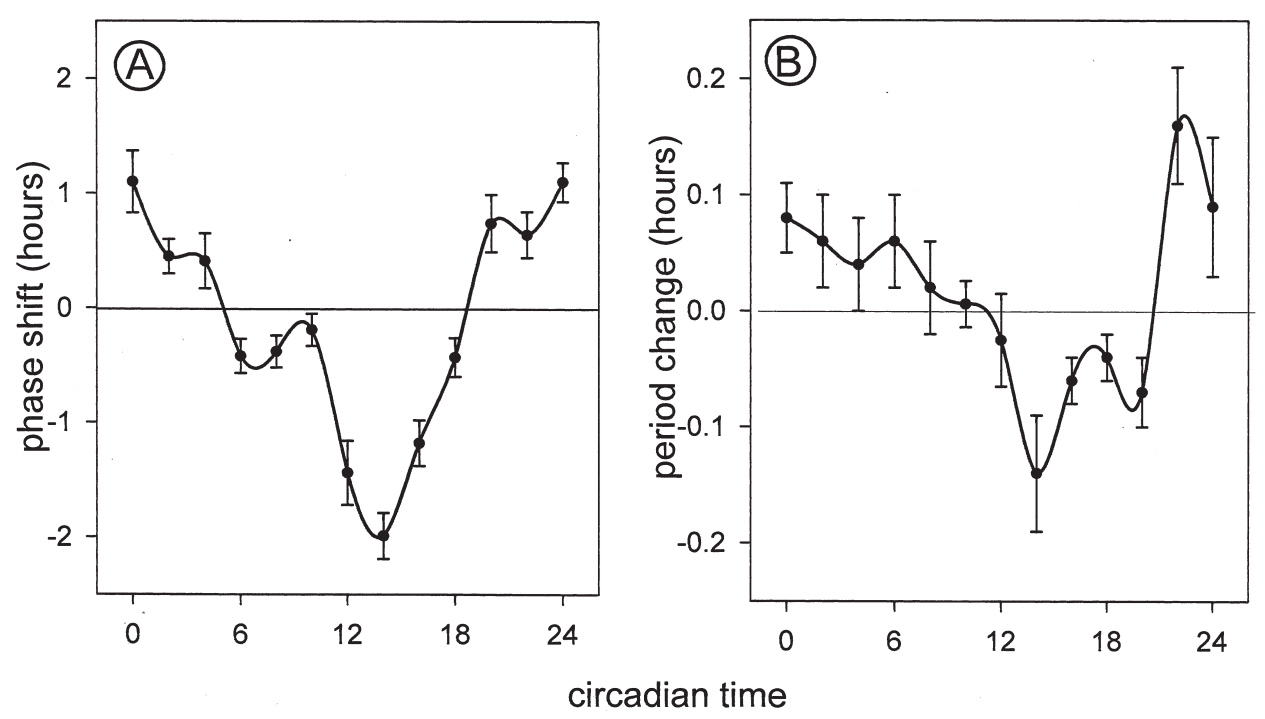

Figure 2. Phase response curve (A) and period response curve (B) constructed for the nocturnal field mouse $M$. booduga, using diffuse daylight pulses of 1000 lux intensity used for $15 \mathrm{~min}$. To estimate phase and period changes, 10-15 animals were used at each phase. Error bars represent standard error around the mean. 


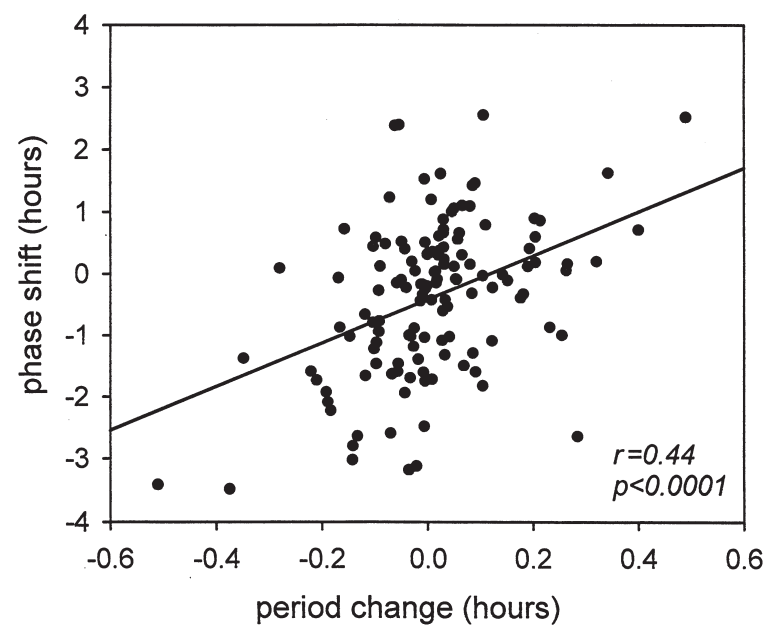

Figure 3. Phase shifts plotted as a function of associated changes in the period in the nocturnal field mouse $M$. booduga. The phase shifts were found to be positively correlated with the period changes $(r=+0.45, p<0.0001 ; n=130)$.

\section{Mus platythrix}

The PRC and $\tau$ RC constructed with $n=69$ individuals for another species of nocturnal rodent $M$. platythrix, using light pulses of strength similar to that used to construct the PRC and $\tau \mathrm{RC}$ for $M$. booduga, and adopting similar protocol, are shown in Fig. 4. The PRC has a waveform similar to that of the M. booduga. As in $M$. booduga, phase shifts in M. platythrix are associated with period changes. One marked similarity between the $\tau$ RCs of these two species of nocturnal rodents is that in both the species, phase advances take place with the shortening of $\tau$, whereas phase delays are associated with the lengthening of $\tau$. The period changes across all the phases showed a small but significant positive correlation with the phase shifts $(r=+0.46, p<0.0001$, Fig. 5).

\section{DISCUSSION}

The $\tau$ RCs for $M$. booduga and $M$. platythrix indicate that these species of nocturnal rodent respond to light pulses in the late subjective night (around ct 2024) by shortening their endogenous period whereas pulses in the early subjective night (ct 14-18) tend to induce increases in circadian cycle length. Period response curves have earlier been published in several diurnal squirrel species, ${ }^{[3,8,9]}$ and in Microtus arvalis. ${ }^{[6]}$ The latter species is not specifically diurnal or nocturnal, but is characterized by ultradian feeding bouts throughout the day and night. In fact, field data suggest that common voles in nature may be predominantly nocturnal in summer and diurnal in winter. ${ }^{[10]}$ In a previous study on Aotus lemurinus, the period response did not depend on the circadian phase at 

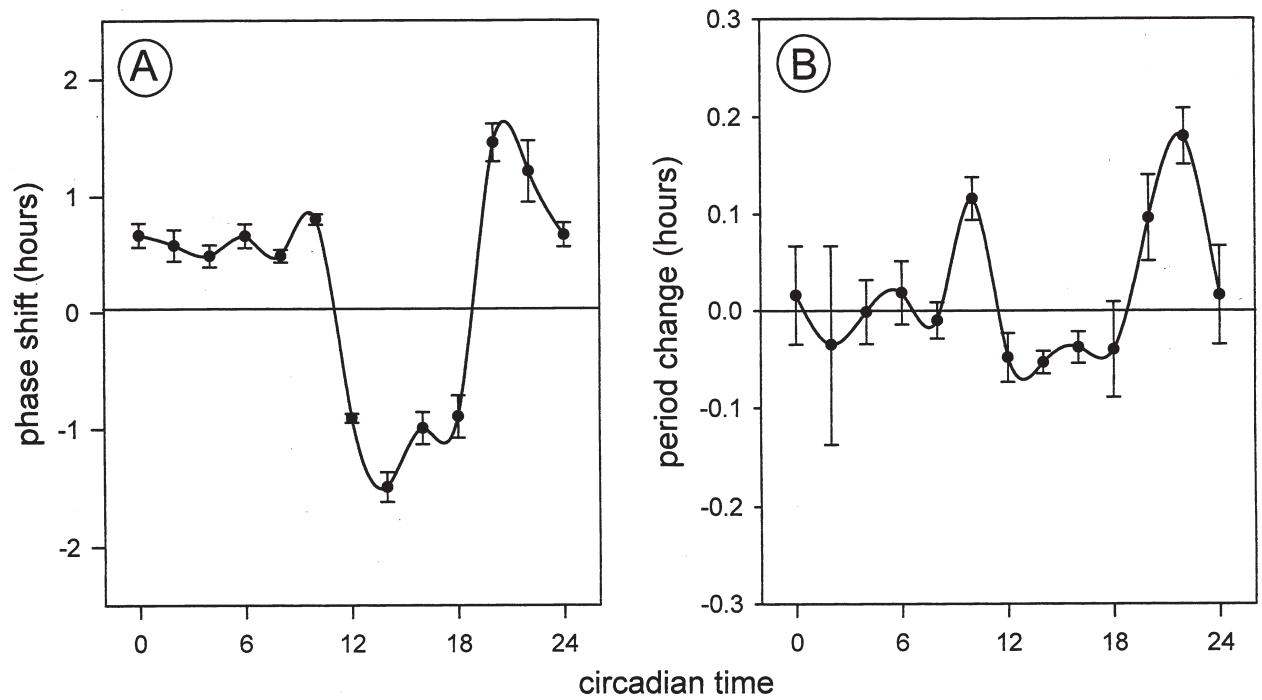

Figure 4. Phase response curve (A) and period response curve (B) constructed for the nocturnal field mouse Mus platythrix, using light pulses of 1000 lux intensity used for $15 \mathrm{~min}$. Six to eight animals were used at each phase to estimate phase and period changes. Error bars represent standard error around the mean.

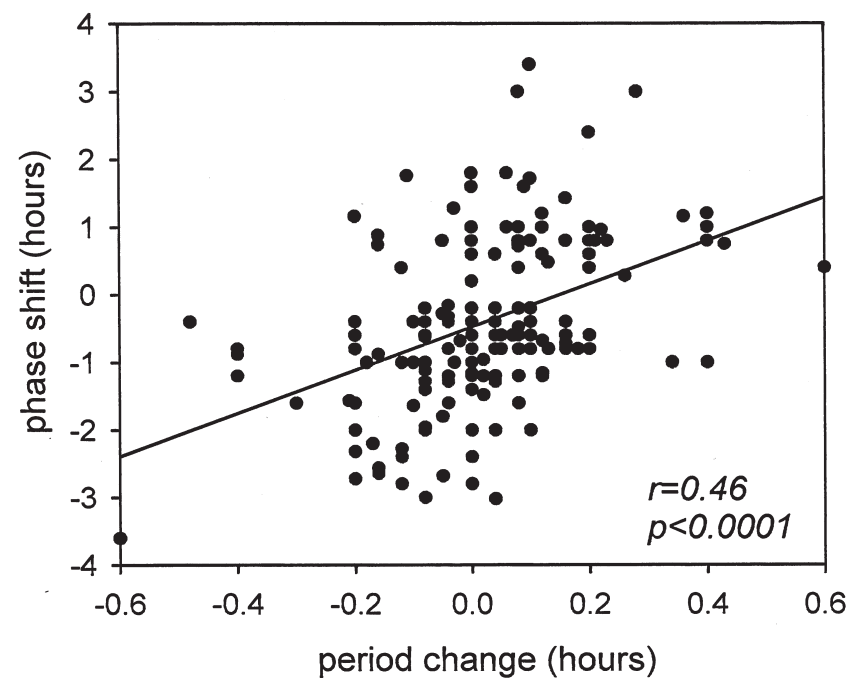

Figure 5. Phase shifts plotted as a function of associated changes in the period in the nocturnal field mouse $M$. platythrix. The phase shifts were found to be positively correlated with the period changes $(r=+0.46, p<0.0001 ; n=69)$. 
which light stimuli was administered. ${ }^{[11]}$ However, in Mus musculas, the correlation between the phase and period responses was similar to those described in the present study. ${ }^{[12]}$ These studies confirm earlier suggestions ${ }^{[2,5]}$ that the circadian system of nocturnal mammals may be characterized by $\tau \mathrm{RC}$ as well as PRCs.

The after-effects of single brief light pulses summarized in the $\tau \mathrm{RC}$ were based on regressions starting from more than $72 \mathrm{~h}$ following each pulse, in order to let transients subside. There is some inherent arbitrariness in the decision where transients end and after-effects start. They may actually be expressions of the same phenomenon. In the M. booduga data, we analyzed the average time course of the deviations of activity onsets - the phase marker used-from the pre-pulse regression (Fig. 1). The result is that there is no evidence for a quadratic component in the explained variance from day 3 following advancing light pulses onwards: the cycle length is no longer changing after this day. Following delaying pulses, the new period length is stable from day 1 onwards. Thus, after-effects are distinct from transients as stable long-term changes in period length following single perturbations of the circadian system.

The shape of the $\tau \mathrm{RC}$ in both species suggests that period lengthening coincides the delaying phase shifts while period shortening coincides with advancing phase shifts. Indeed, the period changes observed in M. booduga at all circadian phases pooled together were positively correlated with the phase shifts (Fig. 3). This is not a trivial result. We are dealing with weak phase resetting, i.e., with stimuli leading to a type-1 PRC. One might presume that light pulses moving the system inside its stable limit cycle (closer to the singularity) would generally speed up its overall motion while pulses moving it outside the stable limit cycle would slow it down. If this were the case, maximal accelerations $(+\Delta \tau)$ would be expected at around ct 18 , the phases during which a single light pulse is known to evoke phase advance and maximal decelerations would be expected during subjective day, during which a single pulse evokes phase delays (gray portion of Fig. 6). This is obviously not the case. The crossover point from deceleration to acceleration is around midnight (ct 18) in most $\tau$ RCs known (summarized in Table 1). There is thus no simple theoretical mechanistic explanation for the shape of the $\tau \mathrm{RC}$.

For a functional explanation, we may turn to a comparison between the $\tau$ RCs of diurnal and nocturnal mammals. Unfortunately, such a comparison is limited by the quality of the data available. The data on all four ground squirrel species summarized by Beersma et al. (Fig. 1 of Ref. [3]), though convincingly demonstrating the existence of $\tau \mathrm{RCs}$, are at best indicative for quantitative purposes. They are based on a limited number of animals and light pulses, and not on thorough quantitative and uniform criteria defining period length. The data set on $M$. arvalis $^{[6]}$ is both substantial and rigidly analyzed, but the species is not strictly diurnal. In Fig. 6, we have summarized the range of values observed as derived from the squirrels represented in Fig. 1 of Ref. [3], along with their average $\tau \mathrm{RC}$. The amplitude of the $\tau \mathrm{RC}$ of both Murid species-based on a 


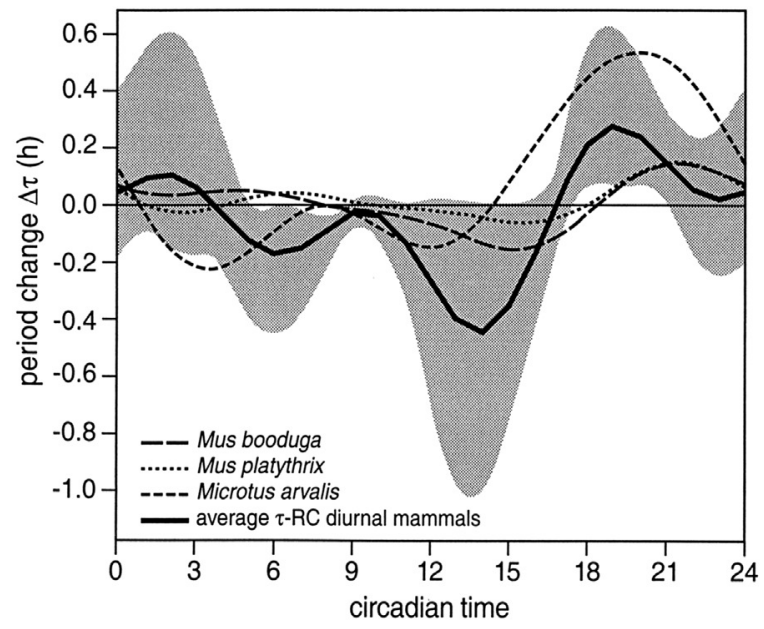

Figure 6. Period response curves constructed for $n=4$ diurnal and $n=3$ nocturnal species constructed using light pulses. The cumulative PRCs for both diurnal and nocturnal animals (obtained by pooling phase shift data at each phases for $n=4$ diurnal and $n=3$ nocturnal species) are shown by the gray area.

harmonic fit with a sine-wave of $24 \mathrm{~h}$ period and two subharmonics (12 and $8 \mathrm{~h})$ appears to be slightly smaller than that of both the diurnal squirrels and the nocturnal/diurnal vole. The minima and maxima of the fitted curves also suggest this. The upward crossing from deceleration to acceleration occurs around ct 1718 , i.e., in the middle of the subjective night, in all cases. Thus, there is no difference in the position of the curves, but at best a difference in amplitude. Downward zero crossings are highly variable (Table 1), probably due to the relative insensitivity to light during the subjective day that is well known from PRCs and has become known as the dead-zone. The amplitude of circadian $\tau$ RCs in nocturnal mammals appears to be smaller than that in diurnal mammals. This may be the reason why although an overwhelming majority of research has been directed at nocturnal mammals, yet $\tau \mathrm{RCs}$ have not so far been reported. More and better data seem to be required to draw a firm conclusion on this issue.

Table 1. Parameters of $\tau$-Response Curves in Different Species of Mammals

\begin{tabular}{lccccc}
\hline Species & Minimum & Maximum & $\begin{array}{c}\text { Upward } \\
\text { Crossing }\end{array}$ & $\begin{array}{c}\text { Downward } \\
\text { Crossing }\end{array}$ & Data Source \\
\hline E. sibiricus & -1.0 & 0.6 & ct 18 & ct 5 & {$[3]$} \\
A. leucurus & -0.2 & 0.1 & ct 17 & ct 0 & {$[9]$} \\
T. striatus & -0.7 & 0.3 & ct 17 & ct 21 & {$[8]$} \\
T. hudsonicus & -0.03 & 0.1 & ct 10 & ct 24 & {$[8]$} \\
M. arvalis & -0.4 & 0.3 & ct 14 & ct 4 & {$[6]$} \\
M. booduga & -0.2 & 0.1 & ct 18 & ct 8 & This paper \\
M. platythrix & -0.1 & 0.1 & ct 18 & ct 10 & This paper \\
\hline
\end{tabular}


The function of period responses is to stabilize entrainment in the face of erratic fluctuations in light intensity. ${ }^{[2,3,13]}$ In their analysis of the contribution of period responses to entrainment accuracy, Beersma et al. ${ }^{[3]}$ have suggested that diurnal mammals would benefit more from such responses at least than burrowing nocturnal rodents. The latter are virtually never exposed to light intensity fluctuations, and indeed would optimally rely more on phase than on period responses. The ratio of the amplitudes of the PRC and the $\tau \mathrm{RC}$, estimated by dividing the maximum (maximum of $\mathrm{PRC} /$ maximum of $\tau \mathrm{RC}$ ) and minimum (minimum of $\mathrm{PRC} /$ minimum of $\tau \mathrm{RC}$ ) of two response curves for diurnal animals, were ca. 6.67 and 6.70, respectively, ${ }^{[3]}$ suggesting that both have a similar waveform. In $M$. booduga and $M$. platythrix, we calculated ratios for the minima ( $\tau$ lengthening/delay) of 25.7 and 19.6, respectively, and for the maxima ( $\tau$ shortening/advance) of 8.7 and 5.7, respectively. This indeed suggests that these two nocturnal rodents depend less on period adjustment than on phase adjustment than the diurnal mammals studied so far, but the difference is especially pronounced for the delay area of the $\mathrm{PRC} / \tau \mathrm{RC}$. More detailed analyses on both nocturnal and diurnal species will be required to conclude that this reflects a general pattern with potential functional meaning.

\section{ACKNOWLEDGMENTS}

We are grateful to Dr. Leela Madhavan for permitting us to use the data on $M$. platythrix from her Ph.D. thesis. We are also grateful to The Norwegian Research Counsel, Norway and University of Groningen, Haren, The Netherlands for supporting travel of VKS to Netherlands. We thank two anonymous referees for their careful reading and constructive suggestions on the manuscript.

\section{REFERENCES}

1. Pittendrigh, C.S. Circadian Rhythms and the Circadian Organization of Living Systems. Cold Spring Harbor Symp. Quant. Biol. 1960, 25, 159-184.

2. Pittendrigh, C.S.; Daan, S. A Functional Analysis of Circadian Pacemakers in Nocturnal Rodents. I. The Stability and Lability of Spontaneous Frequency. J. Comp. Physiol. 1976, 106, 223-252.

3. Beersma, D.G.M.; Daan, S.; Hut, R.A. Accuracy of Natural Entrainment Under Fluctuating Light Conditions: Contributions of Phase and Period Responses. J. Biol. Rhythms 1999, 14, 320-329.

4. Daan, S. Colin Pittendrigh, Jürgen Aschoff, and the Natural Entrainment of Circadian Systems. J. Biol. Rhythms 2000, 15, 195-207.

5. Elliott, J. Circadian Rhythms, Entrainment and Photoperiodism in the Syrian Hamster. In Biological Clocks in Seasonal Reproductive Cycles; Follett, B.K., Follett, D.E., Eds.; Wright: Bristol, 1981; 203-217. 
6. Gerkema, M.; Daan, S.; Wilbrink, M.; Hop, M.; Van der Leest, F. Phase Control of Ultradian and Circadian Rhythms in the Common Vole (Microtus arvalis): The Roles of Light and the Circadian System. J. Biol. Rhythms 1993, 8, 151-171.

7. Sharma, V.K.; Chandrashekaran, M.K.; Nongkynrih, P. Daylight and Artificial Light Phase Response Curves for the Circadian Rhythm in Locomotion Activity of the Field Mouse Mus booduga. Biol. Rhythm Res. 1997, 28, 39-49.

8. Kramm, K.R.; Kramm, D.A. Photoperiodic Control of Circadian Activity Rhythms in Diurnal Rodents. Int. J. Biometeorol. 1980, 24, 65-76.

9. Pohl, H. Characteristics and Variability in Entrainment of Circadian Rhythms to Light in Diurnal Rodents. In Vertebrate Circadian Systems; Aschoff, J., Daan, S., Groos, G., Eds.; Springer: Berlin, 1982; 239-246.

10. Hoogenboom, I.; Daan, S.; Dallinga, J.H.; Schoenmakers, M. Seasonal Change in the Daily Timing of Behaviour of the Common Vole, Microtus arvalis. Oecologia 1984, $61,18-31$.

11. Rappold, I.; Erkert, H.G. Re-entrainment, Phase-Response and Range of Entrainment of Circadian Rhythms in Owl Monkeys (Aotus lemurinus g.) of Different Age. Biol. Rhythm Res. 1994, 25, 133-152.

12. Weinert, D.; Kompauerova, V. Light-Induced Phase and Period Responses of Circadian Activity Rhythms in Laboratory Mice of Different Age. Zoology 1998, $101,45-52$.

13. DeCoursey, P.J. Photoentrainment of Circadian Rhythms: An Ecologist's Viewpoint. In Circadian Clocks and Ecology; Hiroshige, T., Honma, K., Eds.; Hokkaido University Press: Sapporo, 1989; 187-206.

Received December 4, 2001

Returned for revision January 21, 2002

Accepted February 18, 2002 